\title{
Window Based Pulse Shaping Technique for DVB-T System
}

\author{
Mohammed Tarique and Mussab Mohammed Saleh \\ Department of Electrical Engineering, \\ Ajman University of Science and Technology, \\ Fujairah, United Arab Emirates \\ m.tarique@ajman.ac.ae
}

\begin{abstract}
Digital Video Broadcasting (DVB) is an international standard for digital television services. Many versions of DVB are available for commercial use including DVB-H, DVB-S, DVB-C, DVB-T, and DVB-IPTV. In this paper we focus on the performances of DVB-T system. In DVB-T system Orthogonal Frequency Division Multiplexing (OFDM) has been used. The OFDM can minimize Inter Symbol Interference (ISI) and hence it is considered suitable for coping with multipath fading. Like all other OFDM based systems DVB-T signal has some limitations too namely large dynamic range of the signals, sensitivity to frequency error, and high peak to average ratio. It is shown in this paper that the system performance of the DVB-T system highly depends on the pulse shaping filter used in transmitter. Hence, a careful selection of pulse shaping filter is very important for DVB-T system in order to overcome the above mentioned limitations. It is also shown in this paper that the performance of DVB-T system in terms of Bit Error Rate (BER) can be significantly improved provided appropriate pulse shaping filters are chosen.
\end{abstract}

Keywords: DVB-T, OFDM, ISI, transmitter, receiver, filters, pulse shaping, windows, BER, AWGN

\section{Introduction}

Televisions are considered as the most popular electronic devices for entertainment, education, and information. According to the International Telecommunication Union (ITU) the number is TV sets around the World is increasing exponentially (see Fig. 1) and it is expected to reach 700 million by the year 2017 [1]. TV sets were originally developed as an all-electronic system in Europe [2]. Later on a number of color television systems were introduced. Among these systems Sequential Couleur A Memoire (SECAM) and Phase Alternating Line (PAL) became popular. With the advent of digital technology broadcasters and manufacturers started switching analog televisions to digital televisions for two main reasons namely efficient spectrum efficiency and quality of pictures. According to a recent report [1] television sets are now 55\% digital. In the developed world 81 percent of total households with a TV that receive digital signal [3] now-a-days.

The idea of Digital Video Broadcasting (DVB) system originated from the need for converting analog television broadcasting system into digital television broadcasting system. The DVB has been a very popular concept dated back to early nineties. Initially, DVB system was introduced to support stationary signal reception by using rooftop antenna. Extensive researches, carried out in Europe as early as 1997, demonstrated that the DVB system also could support signal reception by using portable devices 
mounted on buses and cars [4]. Since then the DVB system has become a success story in modern broadcasting. The DVB system has drawn considerable attentions of numerous industry-led manufacturers, broadcasters, network operators, and software developers.

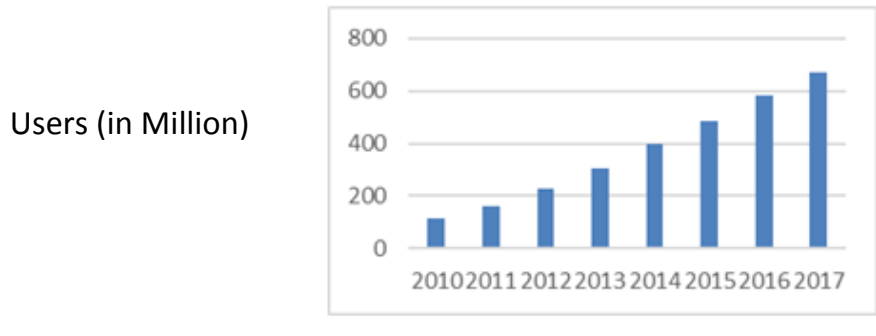

Year

Figure 1 Users of TV around the World

The DVB standard has been introduced, evolved, and maintained by DVB project [5]. Regulatory bodies including European Broadcasting Union, European Committee for Electrotechnical Standardization, and Joint Technical Committee of European Telecommunication Standards Institute are actively involved in the evolution of the DVB system. Many commercial versions of DVB systems are available today. Among these versions DVB-S, DVB-C, and DVB-T are worthwhile to mention here. These versions have been proposed for satellite network, cable networks, and terrestrial networks respectively [6-7]. Some other versions namely DVB-H and DVB-IPTV have been proposed for TV services in handheld devices and Internet Protocol (IP) respectively. In this paper we focus on the DVB-T system.

In DVB-T system the transmitted signal is a Moving Picture Experts Group-Two (MPEG-2) transport system. The DVB-T signal undergoes channel coding and modulation before it is transmitted. In order to enable DVB-T receiver to correct errors a forward error correction (FEC) is used. The DVB-T system has been designed for digital terrestrial television services. It operates within the existing Very High

Table 1: Comparison of " $2 \mathrm{~K}$ mode" and "8k mode' [8]

\begin{tabular}{|c|c|c|c|c|c|c|c|}
\hline \multicolumn{2}{|c|}{ OFDM mode } & \multicolumn{3}{|c|}{$2 \mathrm{~K}$} & \multicolumn{3}{|c|}{$8 \mathrm{~K}$} \\
\hline \multirow{2}{*}{\multicolumn{2}{|c|}{$\begin{array}{l}\text { Number of carriers K } \\
\text { Bandwith of RF channel }\end{array}$}} & \multicolumn{3}{|c|}{$1705(0 . .1704)$} & \multicolumn{3}{|c|}{$6817(0.6816)$} \\
\hline & & $6 \mathrm{MHz}$ & $7 \mathrm{MHz}$ & $8 \mathrm{MHz}$ & $6 \mathrm{MHz}$ & $7 \mathrm{MHz}$ & $8 \mathrm{MHz}$ \\
\hline \multicolumn{2}{|c|}{ Spacing $\mathrm{K}_{0}$ and $\mathrm{K}_{\max }$} & $5,71 \mathrm{MHz}$ & $6,66 \mathrm{MHz}$ & $7,61 \mathrm{MHz}$ & $5,71 \mathrm{MHz}$ & $6,66 \mathrm{MHz}$ & $7,61 \mathrm{MHz}$ \\
\hline \multicolumn{2}{|c|}{ Carrier spacing } & $3348 \mathrm{~Hz}$ & $3906 \mathrm{~Hz}$ & $4464 \mathrm{~Hz}$ & $837 \mathrm{~Hz}$ & $977 \mathrm{~Hz}$ & $1116 \mathrm{~Hz}$ \\
\hline \multicolumn{2}{|c|}{ Duration $\mathrm{T}_{\text {want }}$} & $299 \mu \mathrm{s}$ & $256 \mu \mathrm{s}$ & $224 \mu \mathrm{s}$ & $1195 \mu \mathrm{s}$ & $1024 \mu s$ & $896 \mu \mathrm{s}$ \\
\hline \multirow{4}{*}{$\begin{array}{l}\text { Guard } \\
\text { Interval }\end{array}$} & $1 / 4$ & $75 \mu \mathrm{s}$ & $64 \mu \mathrm{s}$ & $56 \mu \mathrm{s}$ & $299 \mu \mathrm{s}$ & $256 \mu \mathrm{s}$ & $224 \mu \mathrm{s}$ \\
\hline & $1 / 8$ & $37 \mu \mathrm{s}$ & $32 \mu \mathrm{s}$ & $28 \mu \mathrm{s}$ & $149 \mu \mathrm{s}$ & $128 \mu \mathrm{s}$ & $112 \mu \mathrm{s}$ \\
\hline & $1 / 16$ & $19 \mu \mathrm{s}$ & $16 \mu \mathrm{s}$ & $14 \mu \mathrm{s}$ & $75 \mu \mathrm{s}$ & $64 \mu \mathrm{s}$ & $56 \mu \mathrm{s}$ \\
\hline & $1 / 32$ & $9 \mu s$ & $8 \mu s$ & $7 \mu s$ & $37 \mu \mathrm{s}$ & $32 \mu \mathrm{s}$ & $28 \mu \mathrm{s}$ \\
\hline \multicolumn{2}{|c|}{ Carrier modulation } & \multicolumn{6}{|c|}{ QPSK, 16-QAM, 64-QAM } \\
\hline \multicolumn{2}{|c|}{ Inner code rate } & \multicolumn{6}{|c|}{$1 / 2,2 / 3,3 / 4,5 / 6,7 / 8$} \\
\hline
\end{tabular}

Frequency Band (i.e., 50-230 MHz) and Ultra High Frequency Band (i.e., 470-870 MHz). Two modes of operations have been defined for DVB-T standard namely " $2 \mathrm{~K}$ mode" and " $8 \mathrm{~K}$ mode". The " $2 \mathrm{~K}$ mode" has been defined for DVB-T transmission and the " $8 \mathrm{~K}$ mode" has been defined for DVB-H transmission [5]. For limited distance transmission the " $2 \mathrm{~K}$ mode" is considered suitable and for long distance transmission the " $8 \mathrm{~K}$ mode" is preferable. Some of the key features and differences between these two modes are listed in Table 1. 
The DVB standard allows different levels of Quadrature Amplitude Modulation (QAM) as shown in Table 1 and it uses different inner code rates. The system also allows two levels of hierarchical channel coding and modulation. The basic functional block diagram of DVB standard is shown in Fig. 2 . It is shown in the figure that the signal processing functions applied to the input data stream are (i) transport multiplex adaptation, (ii) outer coding, (iii) outer interleaving, (iv) inner coding, (v) inner interleaving, (vi) mapping and modulation, and (vii) OFDM transmission. Most of the signal processing functions as shown in Fig. 2 is done in a digital signal processor. The performance of DVB system highly depends on the modulation as well as the underlying channel conditions. Some basic components of DVB-T system are OFDM, Digital to Analog Converter (D/A), and the front end. In this paper we focus on the digital to analog converter block shown in Fig.2.

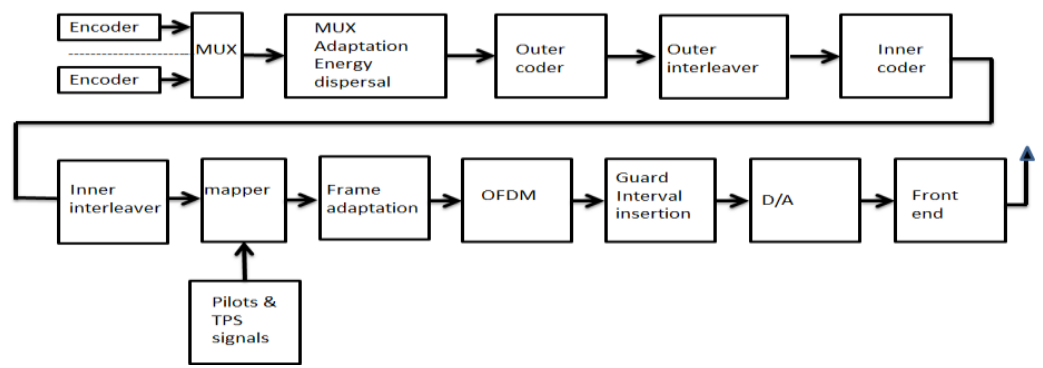

Figure 2 Block Diagram of DVB system

The rest of the paper is organized as follows. Some related works have been presented in section 2 . The system model used in this work is presented in section 3 . The window functions have been described in section 4 . The simulation results are presented in section 5 . The paper has been concluded with section 6.

\section{Related Works}

It has been proven and accepted that OFDM system supports high data speed transmission in mobile environment. It is considered very suitable for selective fading channel [13, 14]. Other advantages of OFDM system include multi-path delay spread tolerance, immunity to frequency selective fading channels, high spectral efficiency $[15,16]$. However, OFDM system has disadvantages too. One of them is the sensitivity of OFDM signal against carrier frequency offset which causes ICI $[9,10,11,12,13,14,15,16]$ and another one is the large variation in envelope of OFDM signal, which causes high peak-to-average power ratio (PAPR) $[9,10]$. Here some of the works that are directly related to our work are presented below.

Pulse shaping of multi carrier signal of OFDM system has been investigated in [17]. Some pulse shapes including Rectangular pulse (REC), Raised cosine pulse (RC), Better than raised cosine pulse (BTRC), Sinc power pulse (SP), and Improved sinc power pulse (ISP) have been considered in [17]. The authors investigated the impulse responses and frequency spectrums of OFDM system for two cases namely without pulse shaping and with pulse shaping. Based on the simulation results the authors concluded that the performance of OFDM system is better with various pulse shapes compared to the same without pulse shape.

In [18] the authors have proposed a semi-blind channel estimation technique for MIMO-OFDM system based on pulse shaping technique. The main objective of the work is to reduce the Inter Symbol Interference (ISI). The pulse shaping filter has been realized by a square root raised-cosine filter. By 
Mohammed Tarique and Mussab Mohammed Saleh; Window Based Pulse Shaping Technique for DVB-T System, Transactions on Networks and Communications, Volume 4 No. 1, February (2016); pp: 25-37

utilizing the knowledge of pulse shaping filters a semi-blind estimation method has been developed. The authors presented some simulation results in the paper. They concluded that the pulse shaping filter has the spectrum with small side lobes. Hence, it leads less Inter Carrier Interference (ICI) and provides better bandwidth efficiency.

A new pulse shaping method for OFDM system called scale alpha has been presented in [19]. In this pulse shaping method alpha is an important parameter and the authors vary the alpha in the range of 0.25 to 1.0. The performance of the OFDM system was compared with other functions namely doublejumped, raised cosine, and Frank pulses. The authors show that the proposed pulse shape performs better than other pulse shapes. The simulated results presented in [19] show that the ICI power can be reduced by almost seven percent by using the scale alpha pulse.

The work presented in [22] is somehow related to our present work. In [20] the authors investigated a number of broadband pulses and narrow band pulses. Mathematical models for each pulse shaping filters have been presented in the work. It has been shown that the square root raised cosine pulse is the best candidate for the OFDM system. It has been shown in [20] that the square root raised cosine pulse not only reduces the peak to average ratio, but also reduces the bit error rate of the system.

Optimized "better-than" raised cosine (BTRC) pulses have been presented in [21] to suppress the ICI effects in OFDM system. The authors came up with some new parameter that follow a quadratic relation with the filter's roll-off factor. The effects of frequency offset have also been addressed by the authors. The simulation results show that BTRC can enhance the performance of the OFDM system in terms of ICI power reduction and Signal to Interference Ratio (SIR).

In our present work we investigate the effects of pulse shaping on the performance of an OFDM system. In this work we choose DVB-T system as our OFDM simulation. In contrast to other related work we focus more on a practical system. We consider a large number of window functions namely Blackman, Blackman-Harris, Gaussian, Hamming, Kaiser, Bohman, Chebyshev, Flattop, Hamming, Hanning, Nuttal, Tukey, Parzewin, Triangle, and Rectangular. These window functions have been widely used in the field of digital signal processing. More specifically these have been widely used in the filter design because of their excellent frequency domain behaviors. In this work we investigated these window functions to improve the performance of DVB-T system.

\section{System Model}

The DVB-T transmitted signals are organized into frame structure. Each frame consists of 68 OFDM symbols [6]. Each symbol consists of a set of K=1705 carriers in the " $2 \mathrm{~K}$ mode" and K=6817 carriers for "8K mode". The transmitted OFDM symbol can be mathematically expressed by

$$
\begin{gathered}
s(t)=\operatorname{Re}\left\{e^{2 \pi f_{c} t} \sum_{m=0}^{\infty} \sum_{l=0}^{67} \sum_{k=K_{\min }}^{K_{\max }} c_{m, l, k} \times \psi_{m, l, k}(t)\right\} \\
\text { Where, } \psi_{m, l, k}(t)=\left\{e^{j 2 \pi \frac{k^{\prime}}{T_{U}}\left(t-T_{G}-l \times T_{S}-68 \times m \times T_{S}\right.} \quad(l+68 \times m) \times T_{S} \leq t \leq(l+68 \times m+1)\right. \\
\psi_{m, l, k}(t)=0 \text { else }
\end{gathered}
$$


where $k=$ the carrier number, $l=$ the OFDM symbol number, $m=$ the frame number, $K=$ the number of transmitted carrier, $f_{c}=$ the central frequency, $k^{\prime}=$ the carrier index related to the center frequency, and $c_{m, l, k}=$ complex symbol for carrier $k$ of the data symbol number $I$ in frame $m$. The $c_{m, l, k}$ values are normalized according to the constellation points of the modulation alphabet used.

The transmitter and the receiver models presented in [8] have been used in this investigation. The basic signal processing steps done in the front end of the DVB-T transmitter are shown in Fig. 3. Since the number of carriers in DVB-T system is 1705 , we consider 1705 4-QAM symbols as the input data to the transmitter model. We use a bandwidth of $7.61 \mathrm{MHz}$. We divide the $7.61 \mathrm{MHz}$ into 1705 sub-carriers. We select 4096-point the IFFT for generating the OFDM symbols. The output carriers of IFFT are then converted into a continuous time signal in the next signal processing step. This step of the signal processing is accomplished by using a Digital to Analog (D/A) filter and a low pass filter denoted as pulse shaping filter in the block diagram of Fig. 3. The pulse D/A filter converts the discrete time carrier output signals into continuous signal. In the next step a low pass filter with a sharp bandwidth is used to change the shape of the pulse. We choose a Butterworth filter of order 13 for this purpose. Finally, the carrier modulation is performed at the last stage. These process of converting a discrete signal into continuous signal is illustrated in Fig. 4. The generated output DVB-T signal is shown in Fig. 5. The figure shows that the DVB signal widely varies in amplitude with respect to time.

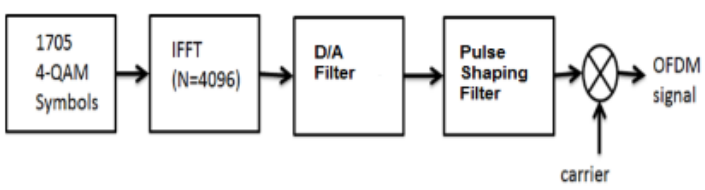

Figure 3 OFDM Transmitter and Receiver model

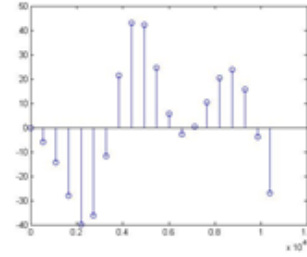

(a) discrete

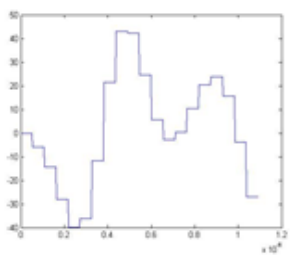

(b) continuous

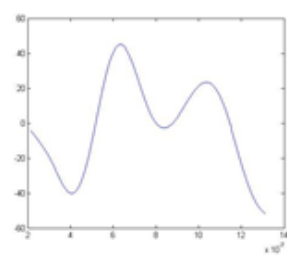

(c) Pulse shaping

Figure 4 Converting discrete OFDM symbol into continuous signal

In this investigation we look for the best D/A filter for DVB-T transmission. Choosing a suitable such filter is import for the DVB-T transmission for the following reasons. The filter has great impact on the power spectral density (PSD) of the DVB-T signal. It helps to produce a compact PSD to reduce Inter Carrier Interference (ICl). It also provides the DBV-T signal with appropriate immunity against noise. Appropriate filter can also reduce the well know problem named high peak to average ratio of the DVB-T signal as shown in Fig. 5. In this investigation we used some popular window functions to change the shape of the DVB-T signals so that the peak to average ration can be reduced and the Bit Error Rate (BER) performance can be improved. The window functions used in this work include Blackman, Blackman-Harris, Gaussian, Hamming, Kaiser, Bohman, Chebyshev, Flattop, Hamming, Hanning, Nuttal, Tukey, Parzewin, Triangle, and Rectangular. The effects on these window functions on the DVB-T system have been investigated in this paper. We primarily investigate the bit error rate (BER) performance of 
the DVB-T system. We also investigate other effect of pulse shaping filters on DVB-T signal's peak-toaverage ratio.

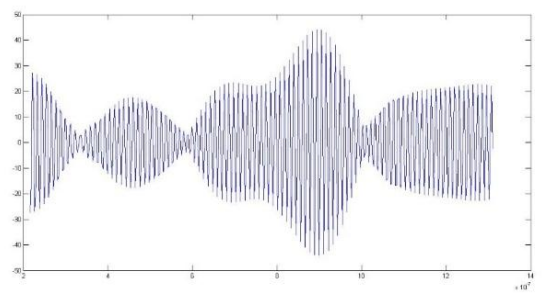

Figure 5 The variation of DVB-T signal with respect to time

\section{Window Functions}

Window functions have been used in the signal processing field for a long time. The most popular applications of the window functions can be found in digital filter design. In this work we consider a number window functions for changing the discrete OFDM signal into continuous signal. We consider a number of window functions as listed in Table 2. Although a vast number of window functions can be found in the literatures [22-23], we consider some popular window functions namely Blackman, Blackman-Harris, Gaussian, Hamming, Kaiser, Bohman, Chebyshev, Flattop, Hamming, Hanning, Nuttal, Tukey, Parzewin, and Triangle in this work. The mathematical expressions of the window function are also listed in Table 2. The impulse response, $h(n)$ and the transfer function, $H(\omega)$ of each of the window function are shown in Table 3(a) and Table 3(b). Although these window functions have drawn considerable attention of the researchers in the field of digital signal processing for designing filters, we consider these windows for shaping the DVB-T signal so that the performance of the same can be improved.

Observing the frequency domain characteristics depicted in Table 3(a) and Table 3(b) we can make the following conclusions. Some window functions have sidelobes, but other window functions do not have sidelobes. For example, Blackman-Harris, Flattop, Nuttal and Parzewin window functions do not have sidelobes. Although these window functions do not exhibit sidelobes, their spectrums decay very slowly. On the other hand window functions like Hamming, Hanning, Kaiser, Tukey, and Triangle exhibit significant sidelobes. In terms of the magnitude of the sidelobes Hamming, Hanning, Kaiser, Bohman, Tukey, and Triangle windows show larger sidelobes. On the other hand Gaussian and Chebyshev window functions have sidelobes with low magnitude. The frequency domain characteristics of the investigated window functions are summarized in Table 4. This table shows that the window functions also widely differ in terms of the location and magnitude of the sidelobes. For example, in Kaiser window function the location of the first sidelobe occurs at lowest normalized frequency (i.e., 0.40). On the other hand, in Blackman window function produces the first sildelobe at the highest normalized frequency (i.e.,0.75). In terms of the magnitude of the sidelobes Tukey window provides the sidelobe of maximum value (i.e., $-20 \mathrm{~dB}$ ). The Kaiser window and Chebyshev window provides the lowest magnitude sidelobe (i.e., -120 $d B)$. 
Table 2 Window functions

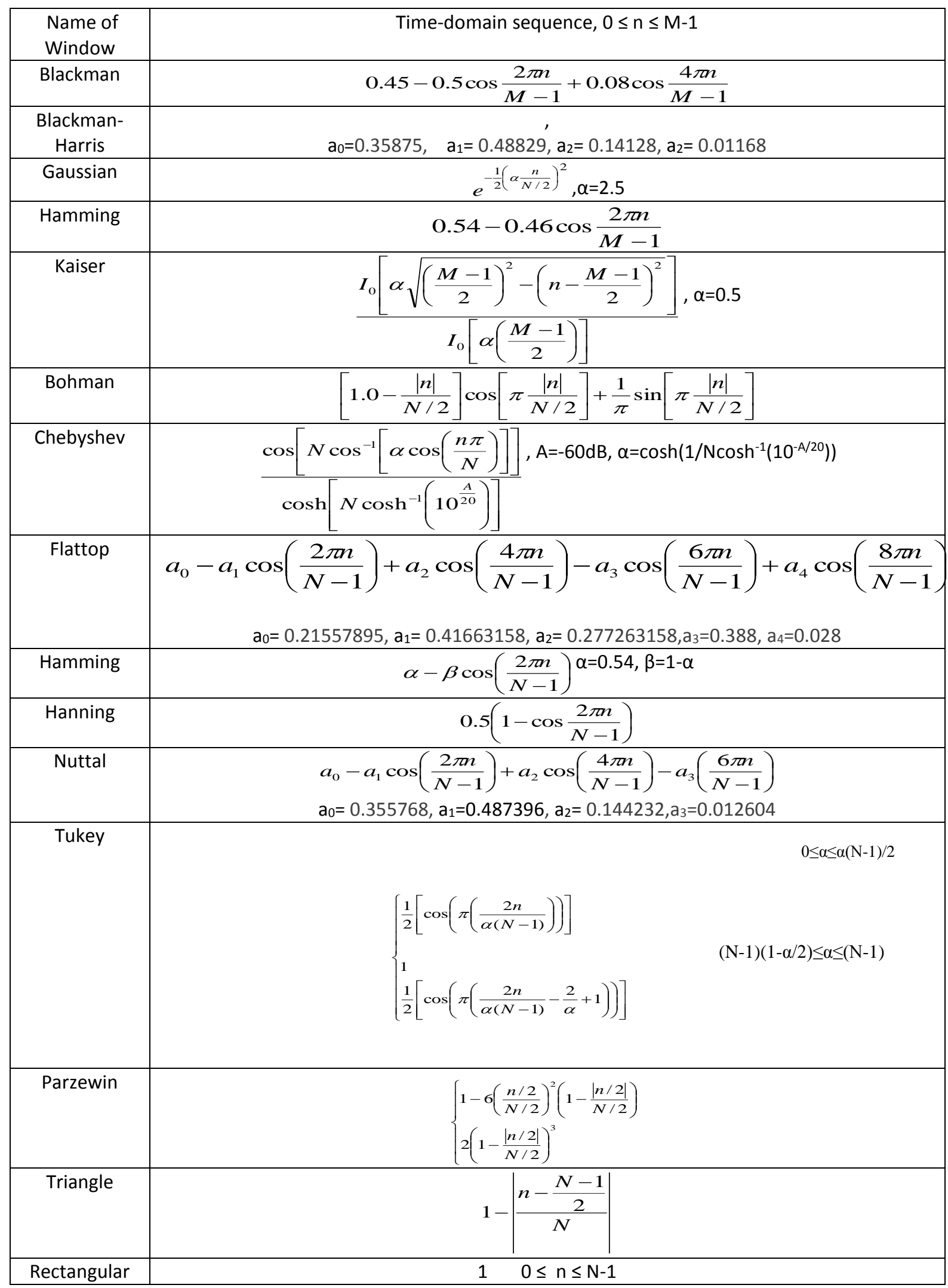


Mohammed Tarique and Mussab Mohammed Saleh; Window Based Pulse Shaping Technique for DVB-T System, Transactions on Networks and Communications, Volume 4 No. 1, February (2016); pp: 25-37

Table 3 (a): Impulse response and spectrum of different Window functions

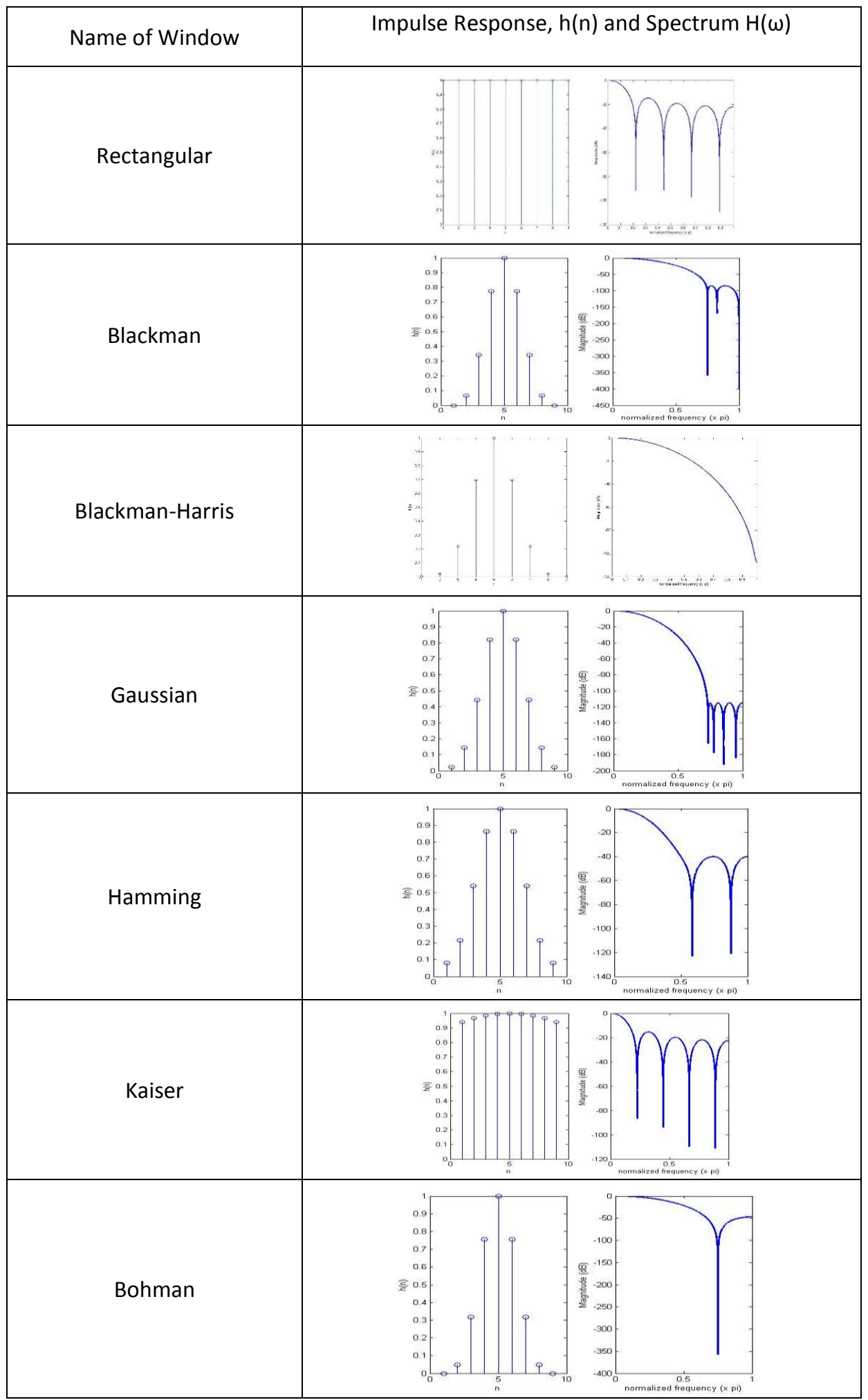


Table 3(b): Impulse response and spectrum of different Window functions

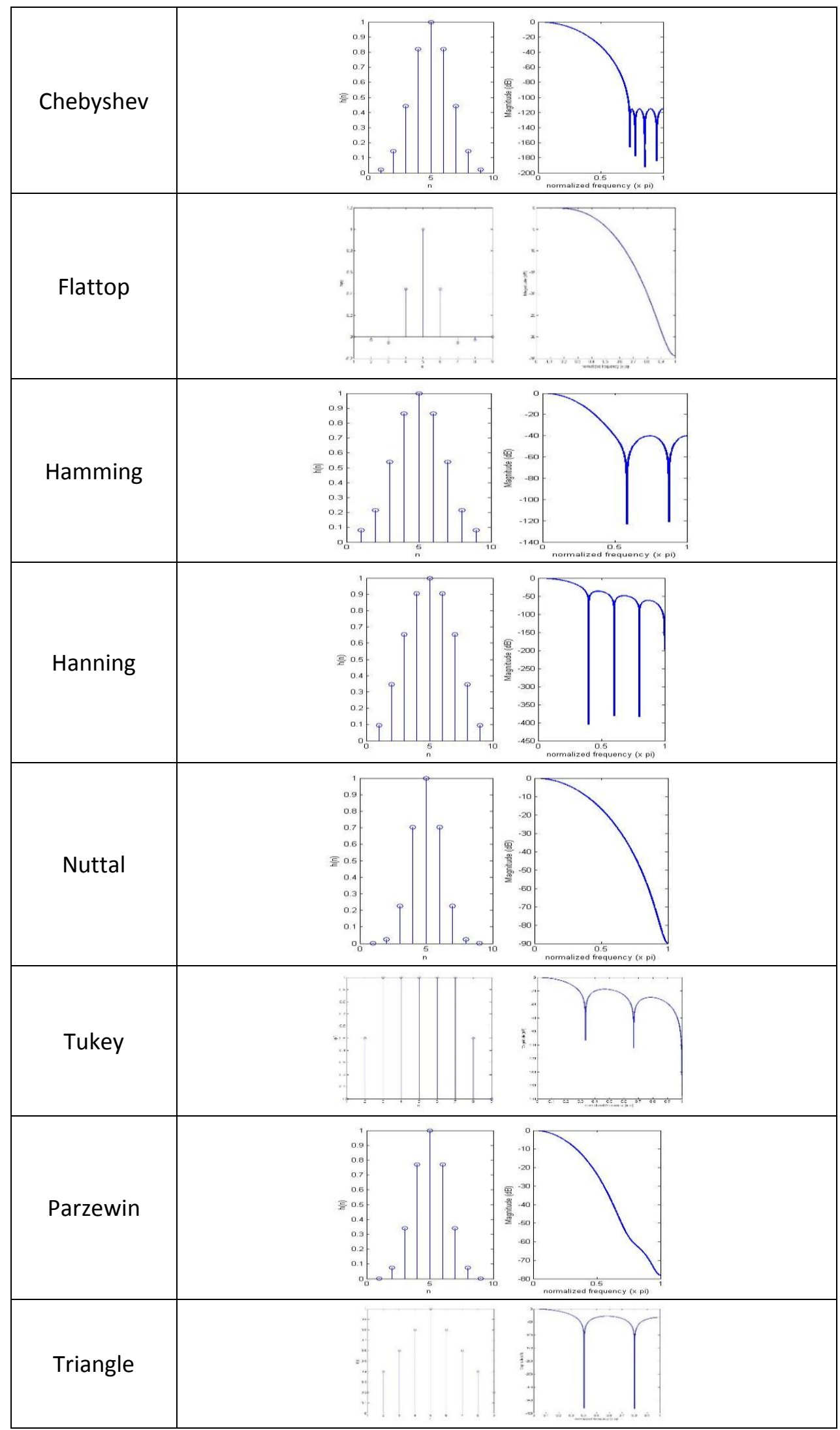


Table 4: Frequency domain characteristics of window functions

\begin{tabular}{|c|c|c|c|}
\hline Window Function & $\begin{array}{c}\text { Location of the first } \\
\text { side lobe } \\
\text { (normalized) }\end{array}$ & $\begin{array}{c}\text { Magnitude of the first side } \\
\text { lobes }\end{array}$ & $\begin{array}{c}\text { Location of the main } \\
\text { lobe termination } \\
\text { (normalized) }\end{array}$ \\
\hline Blackman & 0.8 & $-100 \mathrm{~dB}$ & 0.75 \\
\hline Blackman-Harris & No sidelobe & Not Applicable & Not Applicable \\
\hline Gaussian & 0.75 & $-120 \mathrm{~dB}$ & 0.7 \\
\hline Hamming & 0.65 & $-45 \mathrm{~dB}$ & 0.6 \\
\hline Kaiser & 0.4 & $-20 \mathrm{~dB}$ & 0.25 \\
\hline Bohman & 0.9 & $-50 \mathrm{~dB}$ & 0.7 \\
\hline Chebyshev & 0.65 & $-120 \mathrm{~dB}$ & 0.7 \\
\hline Flattop & No sidelobe & Not Applicable & Not Applicable \\
\hline Hanning & 0.5 & $-50 \mathrm{~dB}$ & 0.48 \\
\hline Nuttal & No Sidelobe & Not Applicable & Not Applicacable \\
\hline Tukey & 0.45 & $-20 \mathrm{~dB}$ & 0.3 \\
\hline Parzewin & No sidelobe & Not Applicable & Not Applicable \\
\hline Triangle & 0.6 & -40 & 0.4 \\
\hline
\end{tabular}

\section{Simulation Results}

The bit error rate of the DVB-T signals for different window functions are shown in Fig. 6(a), Fig. 6(b), and Fig. 6(c). For the clarity of the presentations we splits the simulation results into three figures. Fig.6(a) shows the performances of DVB-T for Hanning, Gaussian, Kaiser, Chebyshev and Blackmann window. This figure shows that Hanning and Kaiser window functions perform the same way and they outperform other window functions namely Chebyshev and Blackmann. Chebyshev window provides the poorest performance.

Figure 6(b) shows the bit error rate performances of DVB-T system for Hamming, Nuttal, BlackmanHarris, Bartlett, and Triangle window functions. It is depicted in Fig.6(b) that Hamming and Triangle windows outperform other window functions. On the other hand the Blackman and Nuttal window functions are not considered suitable for DVB-T system.

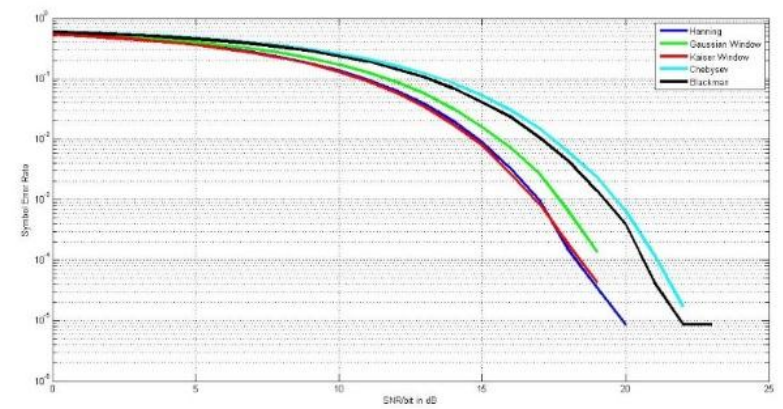

Figure 6 (a) Bit Error Rate (BER) of window functions part I

The performances of DVB-T system for Parzewin, Flattop, Tukey, Rectangular, and Bohman are shown in Fig. 6(c). It is depicted in this figure that Tukey and Rectangular window functions perform better than their other four counterparts. Considering all the performances presented in Fig. 6(a), Fig. 6(b) and Fig. 6(c) we conclude that the Rectangular and Tukey window functions are good candidates for pulse shaping filter in DVB-T transmission. Although Hamming and Hanning window demonstrates similar 
performances, Tukey window and Rectangular window undoubtedly are the best choices for pulse shaping filter in DVB-T transmission.

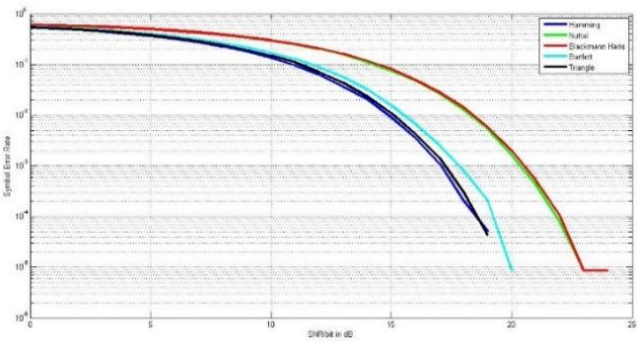

Figure 6 (b) Bit Error Rate (BER) of window functions part II

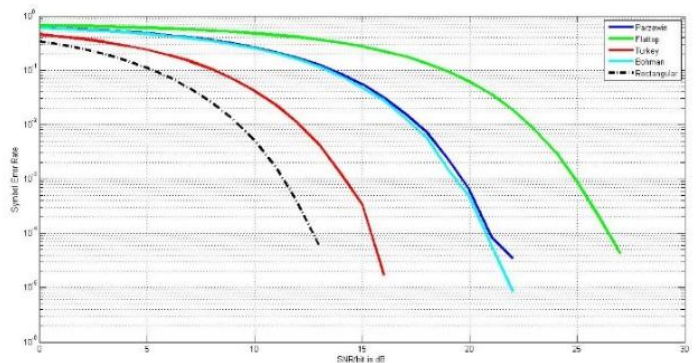

Figure 6 (c) Bit Error Rate (BER) of window functions part III

The effects of filters on amplitude variation of the DVB-T signal with respect to time has also been investigated in this work. Table 5 shows the variations in the amplitude of the DVB-T signals for different window functions. This table lists the average value, maximum value, and the ratio of peak to average value for different window functions. It is depicted in this figure that the low value of the peak to average value is achieved by using either the Tukey window or the Rectangular window.

Based on the simulation results we conclude that among the window functions the Rectangular and Tukey window functions exhibit the low BER for a given signal to noise ratio compare to other window functions. Although the frequency spectrum of the rectangular window shows significant sidelobes as shown in Table 3(a) and Table 3(b), but this window functions provide the best performance in terms of BER. The peak to average ratio as listed in Table 5 shows that the rectangular window is the best choice for DVB signal transmission.

Table 5: The peak-average magnitude of DVB signal for different windows

\begin{tabular}{|c|c|c|c|}
\hline Window & Maximum & Average & Ratio=Maximum/Average \\
\hline Gaussian & 71.0996 & $5.7223 \mathrm{e}-005$ & $1.2425 \mathrm{e}+006$ \\
\hline Kaiser & 145.7600 & $1.2063 \mathrm{e}-004$ & $1.2083 \mathrm{e}+006$ \\
\hline Blackman & 60.2010 & $4.7999 \mathrm{e}-005$ & $1.2542 \mathrm{e}+006$ \\
\hline Chebysev & 57.3238 & $4.5501 \mathrm{e}-005$ & $1.2598 \mathrm{e}+006$ \\
\hline Hamming & 77.7655 & $6.2517 \mathrm{e}-005$ & $1.2439 \mathrm{e}+006$ \\
\hline Hanning & 78.9959 & $6.3514 \mathrm{e}-005$ & $1.2438 \mathrm{e}+006$ \\
\hline Nuttal & 52.1711 & $4.1278 \mathrm{e}-005$ & $1.2639 \mathrm{e}+006$ \\
\hline Blackmanharris & 51.4438 & $4.0914 \mathrm{e}-005$ & $1.2574 \mathrm{e}+006$ \\
\hline Bartlett & 71.2479 & $5.7457 \mathrm{e}-005$ & $1.2400 \mathrm{e}+006$ \\
\hline Triangle & 75.1570 & $6.0731 \mathrm{e}-005$ & $1.2375 \mathrm{e}+006$ \\
\hline Parzewin & 56.5982 & $4.4847 \mathrm{e}-005$ & $1.2620 \mathrm{e}+006$ \\
\hline Flattopwin & 31.0339 & $2.4270 \mathrm{e}-005$ & $1.2787 \mathrm{e}+006$ \\
\hline Tukeywin & 106.9531 & $8.6690 \mathrm{e}-005$ & $1.2337 \mathrm{e}+006$ \\
\hline Bohman window & 58.1022 & $4.5965 \mathrm{e}-005$ & $1.2641 \mathrm{e}+006$ \\
\hline Rectangular & 148 & $1.2371 \mathrm{e}-004$ & $1.2043 \mathrm{e}+006$ \\
\hline
\end{tabular}

We also investigate the performance of DVB-T system by using other popular pulse shaping techniques namely Raised Cosine and Squared Raised Cosine. These pulse shaping techniques have been widely investigated in the literatures $[19,20,21]$. The BER performance of DVB-T system with Raised Cosine Pulse and Squared Raised Cosine Pulse are illustrated in Fig. 7. These performances have been compared 
Mohammed Tarique and Mussab Mohammed Saleh; Window Based Pulse Shaping Technique for DVB-T System, Transactions on Networks and Communications, Volume 4 No. 1, February (2016); pp: 25-37

with other window based pulse shaping technique in the same figure. The simulation results show that window based pulse method provides less BER at a given SNR compared to Raised Cosine and Squared Raised Cosine pulses. This simulation results are somehow contrary to the results presented in $[19,20,21]$.

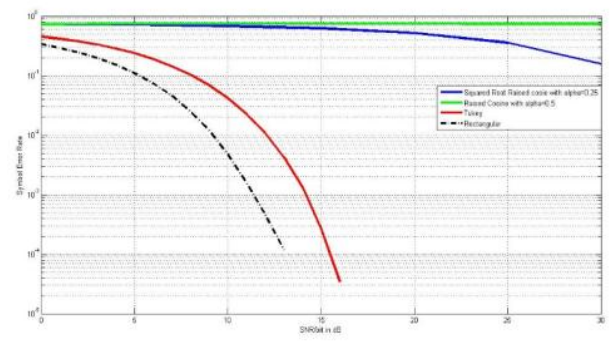

Figure 7 Comparison of window functions with Raised Cosine function

\section{Conclusion}

In this paper the effects of pulse shaping filter on the DVB-T system performances have been investigated. It is shown in this paper that pulse shaping filter used in DVB-T signal is an important element. In order to shape the pulses we consider several window functions. The frequency domain and time domain behaviors of the DVB-T signals have been investigated in this paper. The simulation results show that rectangular window function is the best choice for DVB-T signal transmission. Although rectangular functions exhibits some sidelobes in the frequency domain, this window functions provide the best performance in terms of BER and peak-to-average ratio. Moreover, we have shown in this paper that window based pulse shaping filter outperforms other popular pulse shaping techniques namely the Raised Cosine and the Squared Raised Cosine filters.

\section{REFERENCES}

[1] "The World in 2011: ICT Facts and Figure", Technical Report of International Telecommunication Union (ITU), October 2011

[2] Udelson, J.H., “ The Great Television Race: A History of the American Television Industry", University of Alabama Press, 1982

[3] Press Release of ITU available at http://www.itu.int/net/pressoffice/press_release/2013/

[4] Uwe Ladebusch, and Claudia A. Liss, "Terrestrial DVB (DVB-T): A Broadcast Technology for Stationary Portable and Mobile Use, Procedings of IEEE, Vol. 94, No. 1, January 2006, pp. 183-193

[5] www.dvb.org

[6] Peter S.," DVB: Developing Global Television Standards for Today and Tomorrow", Technical Symposium at ITU, 2011

[7] Reimars, U.H., " DVB-The Family of Internayional Standards for Digital Video Broadcasting”, Proceedings of IEEE, Vol. 4, No. 1, 2006, pp. 173-182 
[8] OFDM Simulation using Matlab available at http://www.ece.gatech.edu/research/labs/ sarl/ tutorials / OFDM/Tutorial_web.pdf

[9] S. D. Assimonis, M. Matthaiou, G. K. Karagiannidis and J. A. Nossek, "Optimized Better Than Raised-TCosine Pulse for Reduced ICI in OFDM Systems", in 17th International Conference on Telecommunications, 2010.

[10] M.Palaivelan, S. Anand and S. P. Venkatesan. 2012. "PAPR and ICI Reduction in OFDM Systems using Modified Raised Cosine Power Pulse Shape", European Journal of Scientific Research ISSN 1450-216X Vol.72 No.4 (2012), pp. 618-627.

[11] Srabani Mohapatra and Susmita Das. 2009. "A New Approach For Performance Improvement of OFDM System Using Pulse Shaping", M.Tech Thesis.NIT Rourkela.

[12] S. Aenagandula, A. Kumar, Srinivas K. and M. Nanda, "Inter Carrier Interference and Signal to Interference Ratio of various Pulse Shaping Functions used in OFDM System with Carrier Frequency Offset", International Journal of Electronics Signals and Systems (IJESS) ISSN: 2231-5969, Vol-1 Iss-3, 2012.

[13] J. Armstrong. "Analysis of new and existing methods of reducing intercarrier interference due to carrier frequency offset in OFDM", IEEE Transactions on Communications, vol. 47, no. 3, pp. 365-369, Mar- 1999.

[14] H.M. Mourad. 2006. "Reducing ICI in OFDM systems using a proposed pulse shape", Wireless Person. Communications. (2006)40: 41-48.

[15] Volkan Kumbasar and O־guz Kucur. 2007. "ICI reduction in OFDM systems by using improved sinc power pulse", Digital Signal Processing 17 (2007) 997-1006.

[16] Srabani Mohapatra and Susmita Das, "Performance Enhancement of OFDM System with ICI Reduction Technique", In Proceedings of the World Congress on Engineering, Vol. I, July, 2009.

[17] D. Gandhi, S. Gupta, and U. Dalal, " Implementing of Pulse Shaping Technique in OFDM system", International Journal of Computer Application, Vol. 68, No. 10, April 2013, pp. 19-23

[18] R. Muthupriya, R. Jeyanthi, and N. Malmurugan, " Semi Blind Channel Estimation Technique with Pulse Shaping for MIMO-OFDM Systems", International Journal of Innovation Research in Science, Engineering, and Technology, Vol. 3, No. 3, March 2014, pp. 1644-1648

[19] Nor Adibah, Rezali Ngah, and Hamza M.R. Al-khafaj, “ Inter-carrier Interefernce Mitigation in OFDM System using a New Pulse Shaping Approach", International Journal of Engineering and Technology, Vol. 6, No. 6, December 2014, pp. 2738-2746

[20] Sultana, T. and Shawkat S.A., " Employment of pulse shaping techniques for efficient PARR reduction in OFDM system", Proceedings of the $17^{\text {th }}$ International Conference on Computer and Information Technology, Dhaka, December 2014, pp. 482-487

[21] Stylianos D. Assimonis, Michail Matthaiou, George K. Karagiannidis, and Josef A. Nossek, “ Optimized Better-Than Raised Cosine Pulse for Reduced ICI in OFDM System", Proceedings of the $17^{\text {th }}$ International Conference on Telecommunications, Doha, April 2010, pp. 249-252

[22] Fredric J. Harris, "On the use of Windows for Harmonic Analysis with the Discrete Fourier Transform", Proceedings of IEEE, Vol. 66, 1978, pp. 51-83

[23] Alber H. Nuttal, " Some Windows with Very Good Sidelobe Behavior", IEEE Transactions on Acoustics, Speech, and Signal Processing, Vol. 29, No. 1, February 1981, pp. 84-91 